\title{
Actualización de la resaca de alcohol Artícullo de revisión
}

Delgado-Sánchez Jonathan Dazaeth*, Herrera-Beiza Bernardo Miguel**, García-Luis Ashley Michelle ${ }^{* * *}$, Herrada-Aguilar Cynthia Jazmín ${ }^{* * * *}$, Lepe-Valdez Sabrina Priscila****, Terrones-Saldívar Ma del Carmen******

\section{- Resumen}

- Introducción: la resaca de alcohol se refiere a la combinación

- de síntomas mentales y fisicos negativos que pueden experi-

- mentarse después de un solo episodio de consumo de alcohol,

- comenzando cuando la concentración de alcohol en sangre

- se acerca a cero. Las resacas generalmente no se consideran

- dañinas y han recibido poca atención científica a pesar de

- ser la morbilidad relacionada con el alcohol más frecuente.

- El objetivo de este trabajo fue revisar el concepto de la re-

- saca ocasionada por el consumo de alcohol, su fisiopatología

- y tratamiento. Metodología: se realizó una búsqueda en la

- literatura de las bases de datos en línea PubMed, EBSCO,

- BVS, Cochrane, Buscador UAA y Epistemonikos utilizando los

- siguientes términos MeSH en español e inglés: "veisalgia", "re-

- saca inducida por alcohol" y "resaca de alcohol". Resultados:

- es necesario diferenciar las resacas en los bebedores sociales

- de los síntomas de abstinencia que experimentan las perso-

- nas con trastornos por consumo de alcohol (es decir, alcoho-

- lismo), lo que no sólo implica el consumo excesivo de alcohol,

- sino que también mantiene un nivel basal de consumo de

- alcohol bastante constante con el consumo continuo durante

- varios días o incluso más. Conclusiones: críticamente, todos los

- modelos de resaca deben basarse en la definición actual de

- resaca de alcohol. En los estudios en sujetos humanos, los

- investigadores necesitan ser objetivos en el diseño de estudio

- que se debe utilizar considerando sus ventajas y limitaciones.

- LUXMÉDICA AÑO 16, NÚMERO 46, ENERO-ABRIL 2021, PP. $29-51$.

- Palabras clave: resaca, resaca de alcohol (RA), veisalgia.

\section{Abstract}

Introduction: Alcohol hangover refers to the combination of negative mental and physical symptoms experienced after a single episode of alcohol use when the blood alcohol concentration approaches zero. Hangovers are generally not considered harmful and have received little scientific attention despite being the most common alcohol-related morbidity. Objective: To review the concept of a hangover caused by alcohol consumption, its pathophysiology, and treatment. Methods: A literature search of the online databases PubMed, EBSCO, BVS, Cochrane, UAA search engine, and Epistemoikos was carried out using the following MeSH terms in Spanish and English: "veisalgia", "alcohol-induced hangover" and "alcohol hangover." Results: Hangover in social drinkers need to be differentiated from withdrawal symptoms experienced by people with alcohol use disorders (alcoholism), which not only involves binge drinking but also maintains a basal level of constant and continuous alcohol consumption for several days or even more. Conclusions: Critically, all hangover models must be based on the current alcohol hangover definition. In studies in human subjects, researchers need to be objective in the study design that should be used considering its advantages and limitations. LUXMÉDICA AÑO 16, NÚMERO 46, ENERO-ABRIL 2021, PP. $29-51$.

*studiante del noveno semestre de la Carrera de Médico Cirujano del Centro de Ciencias de la Salud de la Universidad Autónoma de Aguascalientes, México. Presidente de la Sociedad Aguascalentense de Estudiantes de Medicina 2019-2020. https://orcid.org/0000-0002-9917-9973 Correo electrónico dazaethsanchez@gmail.com

** Estudiante del noveno semestre de la Carrera de Médico Cirujano del Centro de Ciencias de la Salud de la Universidad Autónoma de Aguascalientes, México. Miembro la Sociedad Aguascalentense de Estudiantes de Medicina. https://orcid.org/0000-0002-3310-6958 Correo electrónico berbeiza@gmail.com

*** Estudiante del noveno semestre de la Carrera de Médico Cirujano del Centro de Ciencias de la Salud de la Universidad Autónoma de Aguascalientes, México. Miembro la Sociedad Aguascalentense de Estudiantes de Medicina. https://orcid.org/0000-0001-6652-2539 Correo electrónico ashleyglm@outlook.com

**** Estudiante del noveno semestre de la Carrera de Médico Cirujano del Centro de Ciencias de la Salud de la Universidad Autónoma de Aguascalientes, México. Miembro la Sociedad Aguascalentense de Estudiantes de Medicina. Correo electrónico cindy.herrada17@gmail.com ORCID: https://orcid.org/0000-0001-9156-8475

***** Estudiante del noveno semestre de la Carrera de Médico Cirujano del Centro de Ciencias de la Salud de la Universidad Autónoma de Aguascalientes, México. Miembro la Sociedad Aguascalentense de Estudiantes de Medicina. https://orcid.org/0000-0003-1377-0111 Correo electrónico priscilalepe.pl@gmail.com

****** Profesora investigadora de Tiempo Completo del Centro de Ciencias de la Salud de la Universidad Autónoma de Aguascalientes. https://orcid.org/0000-0002-89062157 Correo electrónico mcterron@correo.uaa.mx

Fecha de recibido:16 de noviembre 2020

Fecha de aceptación: 16 de diciembre 2020

Correspondencia: Dra en C Ma del Carmen Terrones Saldívar. Departamento de Medicina. Edificio 101, planta alta. Centro de Ciencias de la Salud. Universidad Autónoma de Aguascalientes. Avenida Universidad No.940. Código postal 20131. Aguascalientes, Ags. México. Teléfono 4499108433. Correo electrónico mcterron@correo.uaa.mx 


\section{Introducción}

La resaca de alcohol (RA) se refiere a la combinación de síntomas mentales y físicos negativos que pueden experimentarse después de un solo episodio de consumo de alcohol, comenzando cuando la concentración de alcohol en sangre (CAS) se acerca a cero. Algunos de los síntomas comunes son fatiga, dolores de cabeza, náuseas, somnolencia, temblores, debilidad, sed excesiva y sequedad de boca, trastornos del estado de ánimo y apatía. ${ }^{1,2}$ El síntoma característico del RA es el dolor. De hecho, el término médico para la resaca de alcohol "veisalgia" proviene del noruego kveis, que se refiere a la inquietud que sigue al libertinaje, y algia, el término griego para el dolor. ${ }^{3}$

La RA también se ha relacionado con deficiencias neurocognitivas, ${ }^{4}$ un mayor riesgo de accidentes ${ }^{5}$ y una menor productividad (en el lugar de trabajo). ${ }^{6}$ El número de personas que experimentan resaca de alcohol es alto, con estudios que informan un $78 \%$, incluyendo bebedores sociales/ligeros, moderados y fuertes. ${ }^{7}$ Los síntomas de la resaca pueden ser lo suficientemente graves como para perjudicar la rutina diaria, reducir la productividad y causar otras complicaciones asociadas al consumo de alcohol. Por lo tanto, es importante comprender estos síntomas y cómo se relacionan con la gravedad del abuso del alcohol. ${ }^{8}$

La RA como síndrome no se comprende bien y los estudios a menudo han arrojado resultados no concluyentes. Se han propuesto múltiples hipótesis del mecanismo de la $\mathrm{RA}_{1}{ }^{9}$ por mencionar algunas, por un desequilibrio en el sistema inmune, ${ }^{10,11}$ deshidratación, ${ }^{9,12}$ concentración de glucosa en sangre ${ }^{13}$ o por el metabolismo de los congéneres (especialmente el metanol, causa molestias).9,14 Las resacas generalmente no se consideran dañinas y han recibido poca atención científica a pesar de ser la morbilidad relacionada con el alcohol más frecuente y de inducir una serie de consecuencias socioeconómicas como un desempeño laboral deteriorado y una productividad reducida. ${ }^{14,15}$ Es necesario entender la RA para introducirlo ampliamente y con evidencias científicas sólida como evento adverso del consumo del alcohol; por lo que el objetivo de este estudio es realizar una revisión bibliográfica actualizada de la RA.

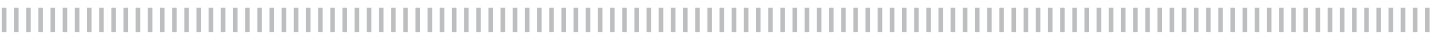

\section{Material y métodos}

Se realizó una búsqueda en la literatura de las bases de datos en línea PubMed, EBSCO, BVS, Cochrane, Buscador UAA y Epistemonikos utilizando los siguientes términos en español e inglés: "veisalgia", "resaca", "resaca inducida por alcohol" y "resaca de alcohol". Los resultados de la búsqueda se limitaron a los estudios publicados en el idioma inglés y español entre el 1 de enero de 2015 y el 23 de mayo de 2020 (no se limitó por diseño de estudio por la escasa información actualizada). En la búsqueda inicial de las 6 bases de datos, encontraron un total de 402 , de los cuales 285 eran duplicados, dejando un total de 
117 artículos únicos. Después de aplicar los criterios de exclusión mencionados en la Fig. 1, se descartaron 30 artículos. De los 87 artículos para esta revisión, se realizó una revisión detallada utilizando las listas de cotejo PRISMA, STROBE, ARRIVE, CONSORT y Narrative Review Checklist, además de un análisis de riesgo de sesgo de las publicaciones seleccionadas. Posterior a esta revisión detallada se excluyeron 32 artículos. Durante el análisis de la información obtenida se agregaron por búsqueda manual de las referencias de los estudios incluidos un total de 21 artículos (Fig. 1).

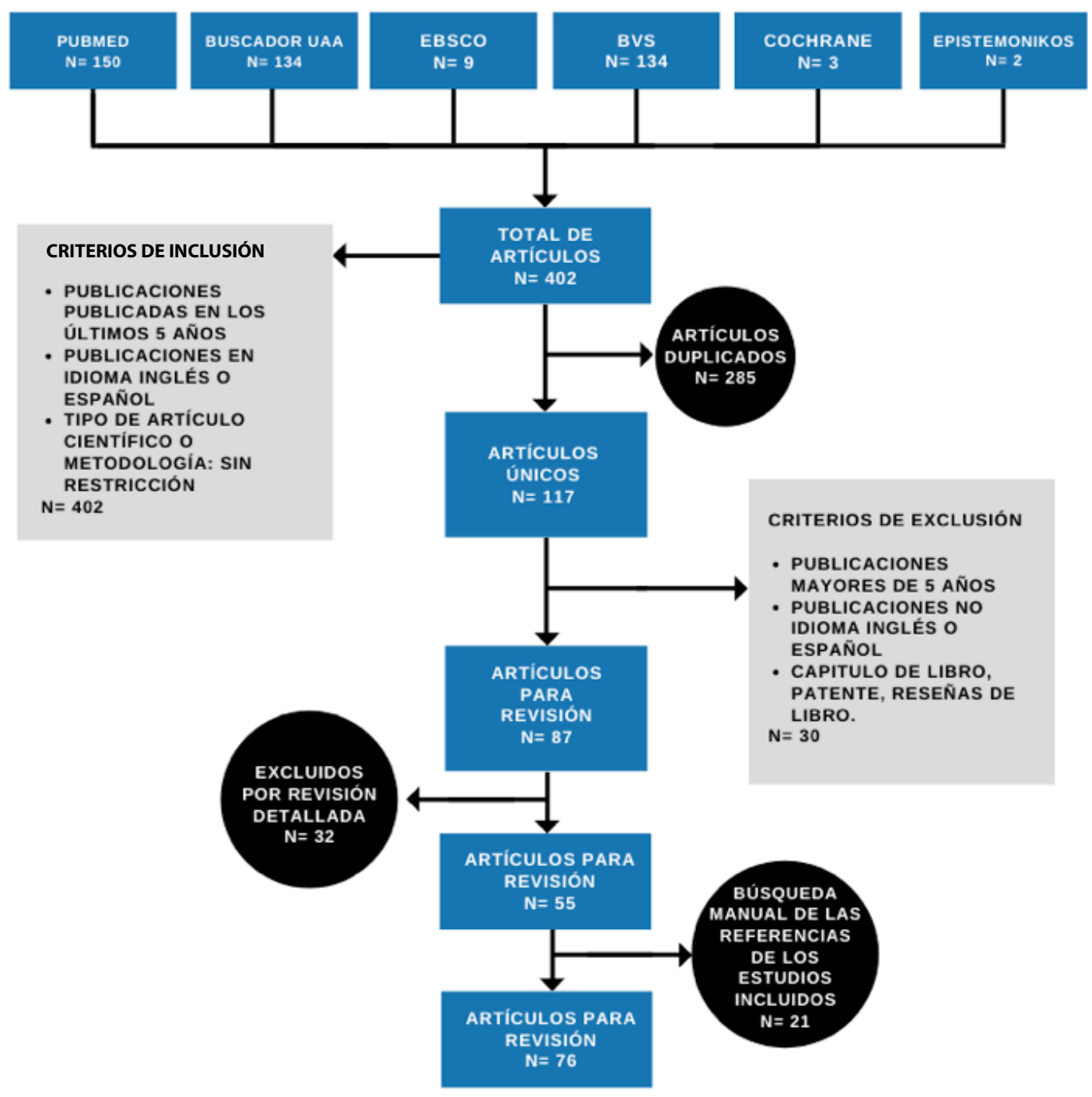

Fig. 1. Resaca de alcohol. Flujograma del proceso de selección de estudios.

\section{SÍNTOMAS}

Los síntomas más comunes son el dolor de cabeza, náusea, cansancio y apatía. ${ }^{16}$ En la gran mayoría de los casos, la RA comienza cuando se despierta después de una tarde o noche de beber, seguido de un período de sueño. ${ }^{17}$ Un estudio del Reino Unido reveló que casi el $20 \%$ de todas las “ocasio- 
nes para beber" se realizaron antes de las 5:00 p.m. ${ }^{18}$ En estos casos, los bebedores pueden experimentar una resaca en la tarde o noche del mismo día. Por lo tanto, la RA se puede experimentar después de un consumo agudo y excesivo de alcohol, independientemente de la hora.

Es necesario diferenciar las resacas en los bebedores sociales de los síntomas de abs- tinencia que experimentan las personas con trastornos por consumo de alcohol (es decir, alcoholismo), lo que no sólo implica el consumo excesivo de alcohol, sino que también mantiene un nivel basal de consumo de alcohol bastante constante con el consumo continuo durante varios días, o incluso más. Penning y cols. ${ }^{19}$ examinó la literatura científica e identificó 47 síntomas de resaca (Tabla 1).

\section{Tabla I}

\section{Síntomas generales de la resaca ${ }^{19}$}

\section{Síntomas de resaca}

\author{
1. Fatiga (estar cansado) \\ 2. Sed \\ 3. Somnolencia \\ 4. Soñolencia \\ 5. Dolor de cabeza (cefalea) \\ 6. Boca seca (xerostomía) \\ 7. Náusea \\ 8. Debilidad \\ 9. Estado de alerta reducido \\ 10. Problemas de concentración \\ 11. Apatía (falta de interés / preocupación) \\ 12. Mayor tiempo de reacción \\ 13. Apetito reducido \\ 14. Torpeza \\ 15. Agitación \\ 16. Vértigo \\ 17. Problemas de memoria \\ 18. Problemas gastrointestinales \\ 19. Mareo \\ 20. Dolor de estómago \\ 21. Temblor \\ 22. Problemas de equilibrio \\ 23. Inquietud \\ 24. Escalofríos
}
25. Transpiración
26. Desorientación
27. Sensibilidad al sonido
28. Fotosensibilidad
29. Afecto embotado
30. Dolor muscular
31. Pérdida del gusto
32. Remordimiento
33. Confusión
34. Culpa
35. Gastritis
36. Impulsividad
37. Sofocos fríos / calientes
38. Vómitos
39. Sensación de latidos fuertes
40. Depresión
41. Palpitaciones
42. Tinnitus
43. Nistagmos
44. Ira
45. Problemas respiratorios
46. Ansiedad
47. Pensamientos suicidas

\section{Tipología temporal de la resaca de alcohol}

La gravedad general de la resaca se evalúa comúnmente utilizando 1 de 3 escalas (tabla 2) utilizadas actualmente: Hangover Symptoms Scale (HSS), The Acute Hangover Scale (AHS) y The Alcohol Hangover
Severity Scale (AHSS). ${ }^{20-22}$ En las 3 escalas, los síntomas individuales se puntúan con la suma o el promedio que representa el puntaje general de gravedad de la resaca. Alternativamente, se pueden usar escalas de ítems individuales que evalúan direc- 
tamente la gravedad general de la resaca. Por ejemplo, un participante califica su resaca general en una escala que va de 0 (ausente) a 10 (extremo), ${ }^{23}$ pero los síntomas pueden tener un efecto significativo y potencialmente sesgo en la calificación global de gravedad de la resaca. Recientemente se ha comparado estas escalas con una escala única de 1 ítem que evalúa la resaca general. Los resultados mostraron que las escalas de síntomas de la resaca subestiman significativamente la gravedad de la resaca (subjetiva), según lo evaluado con una escala general de gravedad de la resaca de 1 ítem. Una posible razón para esto podría ser que la gravedad general de la resaca varía, dependiendo de la frecuencia de aparición de síntomas individuales incluidos en la escala respectiva. En contraste, se puede suponer que, al completar una escala de resaca general de 1 ítem, la calificación incluye todos los síntomas posibles de resaca y su impacto en el funcionamiento cognitivo y físico y el estado de ánimo, lo que refleja mejor la experiencia real de gravedad de la resaca. Por otro lado, depender únicamente de las escalas de síntomas de resaca puede dar falsos positivos en sujetos que informan no tener resaca. Cuando el puntaje promedio de los síntomas es mayor que cero, esto puede llevar a que los sujetos sin resaca se clasifiquen como resaca, ya que muchos de los síntomas de resaca somática y psicológica también se pueden experimentar sin consumir alcohol (por ejemplo, tener dolor de cabeza). ${ }^{24}$ La calificación de gravedad de la resaca general de 1 ítem representa un instrumento de resultado auto informado capaz de medir la gravedad de una afección (es decir, la resaca) o el efecto de un tratamiento en concordancia e incorporación de los tres conceptos, a saber, evaluar la presencia de síntomas, sus efectos sobre la función y su gravedad. Además, como medidas de resultado secundarias (de eficacia), la presencia y gravedad de los síntomas individuales (o su impacto) se pueden evaluar utilizando escalas de síntomas de resaca o individualmente. Sin embargo, no dejan de ser útiles las otras tres escalas.

\section{Tabla 2}

\section{Cuadro comparativo de las escalas de resaca disponibles}

\begin{tabular}{|c|c|c|c|c|}
\hline Escala & Características & Elementos & Interpretación & $\begin{array}{l}\text { Refe- } \\
\text { rencia }\end{array}$ \\
\hline $\begin{array}{l}\text { Hangover } \\
\text { Symptoms } \\
\text { Scale (HSS) }\end{array}$ & $\begin{array}{l}\text { * Evaluar frecuencia } \\
\text { en la que los be- } \\
\text { bedores experi- } \\
\text { mentan síntomas } \\
\text { de resaca en un } \\
\text { año. } \\
\text { * } 13 \text { elementos }\end{array}$ & $\begin{array}{l}\text { 1. "Se sintió extremadamente } \\
\text { sediento o deshidratado" } \\
\text { 2. "Se sintió más cansado de } \\
\text { lo habitual" } \\
\text { 3. "Experimentó dolor de } \\
\text { cabeza" } \\
\text { 4. "Se sintió muy nauseabun- } \\
\text { do". } \\
\text { 5. "Vomitó " } \\
\text { 6. "Se sintió muy débil" } \\
\text { 7. "Tuvo dificultad para con- } \\
\text { centrarse " } \\
\text { 8. "Más sensible a la luz y } \\
\text { sueña más de lo habitual } \\
\text { 9. "Sudaba más de lo habi- } \\
\text { tual" } \\
\text { 10. "Tenía muchos problemas } \\
\text { para dormir " } \\
\text { 11. "Estaba ansioso " } \\
\text { 12. "Se sintió deprimido" } \\
\text { 13. "Experimentó temblores" }\end{array}$ & $\begin{array}{l}\text { * Evaluación dicotómica } \\
\text { (experimentó el sínto- } \\
\text { ma, o no). } \\
\text { * Escala de } 5 \text { puntos: } \\
\text { * Nunca } \\
\text { * } 2 \text { veces o menos (por } \\
\text { año) } \\
\text { * } 3-11 \text { veces (más de } \\
\text { una o dos veces, pero } \\
\text { menos de una vez por } \\
\text { semana) } \\
\text { * } 12-51 \text { veces (más que } \\
\text { una vez al mes, pero } \\
\text { no todas las semanas) } \\
\text { *2 veces o más (una } \\
\text { o más veces por } \\
\text { semana). } \\
\text { * Escala del 0 (ausente) } \\
\text { a } 10 \text { (extremo) para } \\
\text { evaluar severidad por } \\
\text { elemento }\end{array}$ & (21) \\
\hline
\end{tabular}




\begin{tabular}{|c|c|c|c|c|}
\hline $\begin{array}{l}\text { The Acute } \\
\text { Hangover } \\
\text { Scale (AHS) }\end{array}$ & $\begin{array}{l}\text { * Evalúa la severidad } \\
\text { de los síntomas en } \\
\text { un episodio agudo } \\
\text { de resaca } \\
\text { * } 9 \text { elementos }\end{array}$ & $\begin{array}{l}\text { 1. "Resaca" } \\
\text { 2. "Sed" } \\
\text { 3. "Cansancio" } \\
\text { 4. "Dolor de cabeza" } \\
\text { 5. "Mareos / debilidad" } \\
\text { 6. "Pérdida de apetito" } \\
\text { 7. "Dolor de estómago" } \\
\text { 8. "Náuseas" } \\
\text { 9. "Aceleración del corazón" } \\
\quad \text { (palpitaciones) }\end{array}$ & $\begin{array}{l}\text { * Se clasifican en una } \\
\text { escala que varía de } \\
0 \text { a } 7 \\
\text { o Ninguno (0) } \\
\text { o Leve (1-3) } \\
\text { o Moderado (4-6) } \\
\text { o Incapacitante (7) } \\
\text { * La gravedad general } \\
\text { de la resaca se calcula } \\
\text { partiendo del prome- } \\
\text { dio } \\
\text { * Anotar en los nueve } \\
\text { ítems de AHS }\end{array}$ & (20) \\
\hline $\begin{array}{l}\text { The } \\
\text { Alcohol } \\
\text { Hangover } \\
\text { Severity } \\
\text { Scale } \\
\text { (AHSS) }\end{array}$ & $\begin{array}{l}\text { * Evalúa la severidad } \\
\text { de los síntomas de } \\
\text { la resaca } \\
\text { * Se compone de } 12 \\
\text { elementos }\end{array}$ & $\begin{array}{l}\text { 1. "Fatiga" } \\
\text { 2. "Torpeza" } \\
\text { 3. "Mareos" } \\
\text { 4. "Apatía" } \\
\text { 5. "Sudoración" } \\
\text { 6. "Temblores" } \\
\text { 7. "Confusión" } \\
\text { 8. "Dolor de estómago" } \\
\text { 9. "Náuseas" } \\
\text { 10. "Problemas de concentra- } \\
\text { 11. "Palón" } \\
\text { 12. "Sed" }\end{array}$ & $\begin{array}{l}\text { * La gravedad de los } \\
\text { síntomas para cada } \\
\text { elemento se puede } \\
\text { clasificar en una escala } \\
\text { que varía de } 0 \text { (ausen- } \\
\text { te) a } 10 \text { (extremo). La } \\
\text { gravedad general de } \\
\text { la resaca es el puntaje } \\
\text { promedio en los } 12 \\
\text { ítems }\end{array}$ & (22) \\
\hline $\begin{array}{l}\text { Patient- } \\
\text { Reported } \\
\text { Outcome } \\
\text { Measures } \\
\text { (PROMs) }\end{array}$ & * Escala de 1 ítem & 1. Resaca & $\begin{array}{l}\text { * La calificación incluye } \\
\text { todos los síntomas } \\
\text { posibles de resaca y su } \\
\text { impacto en el funcio- } \\
\text { namiento cognitivo y } \\
\text { físico y el estado de } \\
\text { ánimo, lo que refleja } \\
\text { mejor la experiencia } \\
\text { real de la gravedad de } \\
\text { la resaca }\end{array}$ & (24) \\
\hline
\end{tabular}

Verster y cols. ${ }^{25}$ sugieren tres patrones de resaca dominantes: una resaca de disminución continua (resaca de gravedad tipo $1,54.5 \%)$, una resaca de estado estable (resaca de gravedad tipo 2, 19.1\%) y una resaca de curva en forma de $U$ invertida (resaca de gravedad tipo 3, 21.8\%). De estos tres patrones, la resaca tipo 2 de gravedad se asocia con un consumo significativamente menor de alcohol y con los puntajes de gravedad más bajos de los síntomas individuales de la resaca. Las resacas de tipo 1 de gravedad se asocian con la mayor gravedad de los síntomas individuales de resaca. En línea con los niveles significativamente más bajos de consumo de alcohol, las resacas tipo 2 se observaron significativamente más a menudo en mujeres que en hombres. La resaca tipo 1 de gravedad fue significativamente más común en hombres que en mujeres. Las resacas de tipo 3 de gravedad, caracterizadas por la mayor presencia de problemas gastrointestinales, se experimentaron igualmente en hombres y mujeres.

\section{Factores de riesgo y posibles causas}

Algunos autores refieren como factores de riesgo para $\mathrm{RA}$, pero sin limitarse, al consumo excesivo de alcohol, tabaquismo, actividades durante la bebida (ej. bailar o estar en un bar) y el estado emocional durante el consumo de alcohol. Sin embargo, hay bebedores que casi nunca experimentan resaca. ${ }^{17,18}$ Una de las muchas razones potenciales para esto podría ser la 
metabolización del alcohol y/o su metabolito acetaldehído en el hígado. El alcohol se metaboliza principalmente en el hígado a través de una reacción de dos pasos. ${ }^{26}$ Primero, el etanol se oxida en acetaldehído, que es altamente tóxico. Este proceso oxidativo es facilitado por la enzima alcohol deshidrogenasa $(A D H)$, que está presente en alta concentración en el citosol de los hepatocitos. Aunque el primer paso en el metabolismo del alcohol es reversible, el acetaldehído en general se metaboliza rápidamente. En este segundo paso, el acetaldehído ingresa a las mitocondrias donde se oxida en acetato y agua. Este proceso es facilitado por la enzima aldehído deshidrogenasa mitocondrial (ALDH). Para ambos pasos, nicotinamida adenina dinucleótido $\left(\mathrm{NAD}^{+}\right)$es esencial para proporcionar la energía necesaria para la conversión, que se convierte en disponible cuando $\mathrm{NAD}^{+}$se convierte en $\mathrm{NADH}+\mathrm{H}^{+}$. $\mathrm{NADP}^{+}$se puede formar a partir de $\mathrm{NAD}^{+}$, y difiere de $\mathrm{NAD}^{+}$en presencia de un adicional grupo fosfato. ${ }^{27}$ La conversión de acetaldehído en acetato y agua es similar a la que se sirve en exceso en la ruta principal del metabolismo del alcohol y requiere $\mathrm{NAD}^{+}$. Una tercera vía menor oxida el etanol en acetaldehído a través de la catalasa. ${ }^{28}$ En conjunto, las vías oxidativas representan más del $90 \%$ de la eliminación del alcohol. ${ }^{29}$

La variación genética en los alelos para ADH y ALDH puede explicar las variaciones en la respuesta de la resaca al consumo de alcohol. El acetato es un precursor del acetil CoA que se puede convertir en dióxido de carbono y agua en el ciclo de Krebs. Aunque el acetaldehído es altamente tóxico, su rápida oxidación por la ALDH mitocondrial puede impedir cualquier toxicidad, aunque se desconoce su tasa de eliminación en el cerebro.

\section{Relación entre la concentración de alcohol y la resaca}

Con respecto a los diferentes tipos de bebidas alcohólicas, se ha informado que, con la misma cantidad de alcohol consumida, las resacas suelen empeorar después de consumir licores en comparación con el vino o la cerveza. Se puede encontrar una explicación para esto en la variación de la presencia o ausencia de los llamados congéneres. Los congéneres son compuestos que se producen naturalmente en las bebidas alcohólicas como resultado de los procesos de destilación y fermentación. Pueden contribuir al sabor, el aroma y el color de las bebidas alcohólicas. La noción de que el contenido de congéneres difiere entre los espíritus destilados y otras bebidas alcohólicas no es nueva. ${ }^{30}$ La lista de congéneres incluye aminas, amidas, acetonas, acetaldehído, polifenol, metanol, histaminas, aceite de fusel, ésteres y taninos, aunque es limitado el conocimiento que se tiene de cada componente y su relación con la RA. ${ }^{31}$ El metanol y etanol son considerados unos de los compuestos que más pueden contribuir significativamente a la resaca.

La concentración de metanol difiere significativamente entre las bebidas alcohólicas. La eliminación del metanol del cuerpo coincide con el inicio de la resaca de alcohol. Los metabolitos del metanol, el formaldehído y el ácido fórmico, son altamente tóxicos para el cuerpo humano y, por lo tanto, pueden contribuir a experimentar síntomas de resaca. ${ }^{30,32}$ La afinidad de la ADH es mucho mayor por el etanol que por el metanol. Por lo que la concentración de metanol en la orina no se correlaciona significativamente con la gravedad general de la resaca $(p=0.948)$, ni con ninguno de los síntomas individuales de la resaca. ${ }^{21}$

El etanol $(\mathrm{EtOH})$ en la sangre afecta el cerebro y otros tejidos hasta que el hígado lo metaboliza a través de la oxidación, desintoxicando la sustancia y volviéndola inofensiva para los tejidos y órganos. Una pequeña cantidad de EtOH se excreta sin cambios a través de los pulmones y la orina. La velocidad a la que el hígado puede metabolizar EtOH depende de la cantidad 
de alcohol deshidrogenasa. Si la tasa de consumo de EtOH supera la tasa de metabolismo de $\mathrm{EtOH}$, se produce un aumento excesivo en la concentración de alcohol en sangre debido a la cinética de orden cero. Factores que podrían influir en la tasa de absorción de EtOH y el metabolismo durante tales estudios de alcohol, incluyen el consumo de alimentos, así como el sexo del individuo. ${ }^{33}$

A lo largo de la historia de la investigación de la RA, no se ha definido la cantidad exacta de alcohol que se necesita ingerir para desencadenar una resaca. ${ }^{34}$ Verster y cols. ${ }^{12}$ encontraron que sujetos $(n=176)$ que consumieron una media (DE) de 3.0 (1.8) bebidas alcohólicas (10 g de etanol cada una), informaron una gravedad considerable de la resaca a la mañana siguiente, es decir, un puntaje promedio (DE) de severidad de la resaca global de 4.6 (2.4) en una escala de 0-10. A pesar de esto, su CAS máximo estimado fue de $0.03 \%$. Estas observaciones no son consistentes con el consumo excesivo de alcohol en la definición de la resaca de alcohol, ni con el umbral de consumo excesivo de alcohol sugerido de $0.11 \%$ por el Instituto Nacional Sobre Abuso de Alcohol y Alcoholismo (NIAAA) en EUA. ${ }^{35,36}$ Mientras que, en encuestas realizadas en grandes muestras de estudiantes de Canadá $(n=5540)$ y los Países Bajos $(n=6002)$, informaron RA en todos los niveles de CAS. ${ }^{11,37}$ Por lo tanto, las resacas pueden ocurrir a cualquier nivel razonable de CAS, y no se limitan a beber "en exceso" solamente. Debido a esta evidencia, el Grupo de Investigación de Resaca de Alcohol (Hangover Research Group $^{38}$ emitió que el valor umbral de $0.11 \%^{34}$ como criterio para tener resaca debe ser abandonado. ${ }^{12}$

En relación con la concentración de alcohol en el aliento, Mackus y cols. no identificaron una correlación entre el contenido de alcohol en el aliento y la gravedad de la resaca en individuos sensibles a la resaca o insensibles a ésta. ${ }^{11}$ Curiosamente, en orina los niveles de etanol fueron significativamente elevados en sujetos sensibles a la resaca en comparación con la insensibilidad a la resaca el día después del consumo de etanol, que se correlacionó con una variedad de síntomas de resaca. ${ }^{32}$

\section{Lesión hepática como marcador de severi- dad de la resaca}

Vatsalya y cols. ${ }^{7}$ investigaron la relación entre la cantidad/patrones de consumo de alcohol y la sintomatología de la resaca por las categorías de consumo de alcohol. En este estudio participaron hombres y mujeres en tres grupos: 12 bebedores excesivos (BE; $>15$ bebidas / semana); 17 bebedores moderados (BM; 5-14 bebidas / semana); y 12 controles sanos (bebedores sociales/ ligeros, BS; <5 bebidas / semana). Se empleó la prueba de identificación de trastornos por consumo de alcohol (AUDIT, por sus siglas en inglés) y la AHS. El puntaje promedio de AHS mostró incapacidad moderada, y las medidas individuales y los dominios del AHS fueron significativamente elevados en HD en comparación con MD. El puntaje AUDIT mostró efectos principales significativamente altos con los síntomas del dominio mental (resaca, mareos y sensación de hambre) ( $p \leq 0.001$ ), así como el dominio físico (sed, cansancio y dolor de cabeza) ( $p \leq 0.001$ ) en este grupo. Los síntomas del dominio gastrointestinal (náuseas, pérdida de apetito y dolor epigástrico) mostraron efectos principales significativos, aunque moderados, con puntajes AUDIT ( $p \leq 0.001)$ en estos bebedores fuertes. Los síntomas de tres dominios de la AHS (mental, gastrointestinal y simpático) mostraron un aumento significativo específico de dominio en HD. Una relación específica de dominio estuvo presente entre AUDIT y medidas específicas de puntajes de AHS en HD, específicamente con los síntomas de dependencia. La exacerbación en la sintomatología de la resaca podría ser un marcador de trastorno por consumo de alcohol más severo. Los 
grandes bebedores con enfermedad hepática alcohólica mostraron signos tanto de rango clínico de lesión hepática como de marcadores de función. Se sospecha que el papel de la función/lesión hepática en la sintomatología de la resaca es que contribuye a nivel subóptimo del metabolismo del alcohol y metabolitos del alcohol. Esto podría conducir a mayores concentraciones de alcohol en la sangre y a duracio- nes más largas en el sistema. El aldehído, un metabolito del metabolismo del alcohol principalmente en el hígado, tiene efectos tóxicos en el sistema que conducen a patologías (estrés oxidativo), y esto puede estar relacionado con manifestaciones psiquiátricas (abstinencia/resaca). En la figura 3 se ve la posible relación por el consumo excesivo de alcohol mediado por la función hepática.

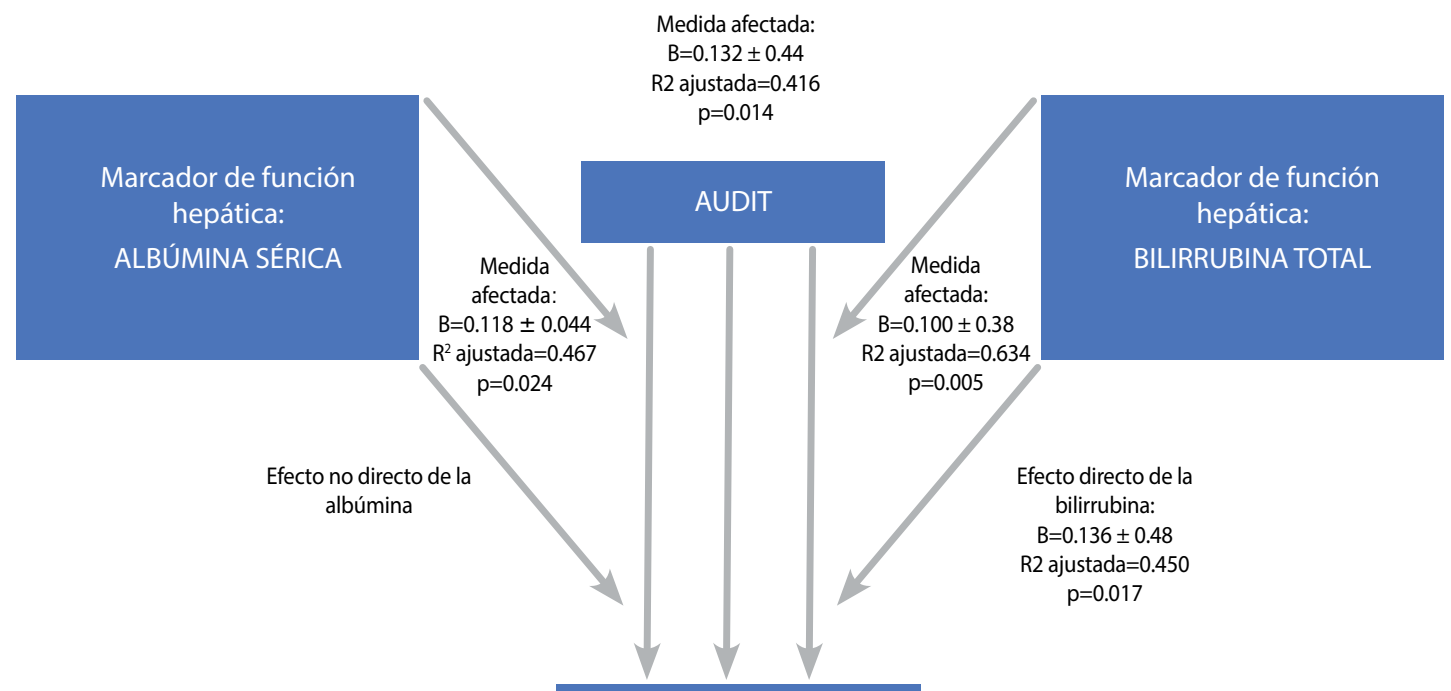

Escala promedio de resaca

Fig. 3. Esquema de los efectos del estado del hígado (insuficiencia hepática) y la gravedad de la bebida en la sintomatología de la resaca. Los marcadores de la función hepática, la albúmina sérica y la bilirrubina total en suero mostraron posibles funciones mediadoras en los efectos de AUDIT sobre los síntomas de la resaca?

Aparentemente esta relación observada podría dar respuestas de los diferentes resultados entre la población que es susceptible a la resaca de aquellos que son resistentes. Sin embargo, en otro estudio en el que se evaluó el metabolismo del alcohol no se encontraron diferencias significativas en CAS a lo largo del tiempo entre el grupo resistente a la resaca y el grupo sensible a la resaca. ${ }^{39}$ Por lo tanto, en contraste con las diferencias en la gravedad de los síntomas observadas en el estado de resaca los hallazgos actuales sugieren que los bebedores resistentes a la resaca son igualmente sensibles a los efectos agudos del alcohol que los bebedores que generalmente experimentan resaca. ${ }^{23}$ Sin embargo, se necesitan investigaciones adicionales sobre los roles de la ADH y el ALDH con respecto a la sintomatología de la resaca que podría ayudar a definir esta relación, en el que su metodología se tome en cuenta los patrones de consumo de alcohol y la sensibilidad a la RA de los sujetos de estudio. 
Ingesta de nutrientes en la dieta y gravedad de la resaca

Se ha demostrado que varios componentes de la dieta influyen en el metabolismo del alcohol y, por lo tanto, pueden afectar el desarrollo de una resaca. De la literatura, es evidente que el ácido nicotínico y el zinc en la dieta juegan un papel fundamental en la oxidación del etanol en acetaldehído. ${ }^{27}$ El zinc es esencial en la conversión de etanol en acetaldehído ${ }^{40}$ y el ácido nicotínico y sus equivalentes son las fuentes dietéticas de $\mathrm{NAD}^{+}$y $\mathrm{NADP}^{+}$, que en conjunto catalizan el metabolismo del alcohol. ${ }^{41}$

La ingesta dietética de estos micronutrientes es necesaria, ya que el cuerpo no puede sintetizarlos por sí mismo. Por lo que se ha estudiado la asociación de la ingesta dietética de ácido nicotínico y zinc con la gravedad de la resaca. Los datos muestran que la ingesta dietética de ácido nicotínico y zinc se asociaron de manera significativa y negativa $(p<0.002)$ con la gravedad general de la resaca. La ingesta dietética de zinc también se asoció de manera significativa y negativa con la gravedad del vómito ( $p<0.002$ ). Esto sugiere que los bebedores sociales que tienen una mayor ingesta dietética de ácido nicotínico y zinc informan resacas significativamente menos graves. Como los bebedores resistentes a la resaca y sensibles a la resaca tenían una ingesta similar de nutrientes en la dieta, la afirmación de ser resistente a la resaca debe basarse en otros factores biopsicosociales desconocidos. ${ }^{27}$

\section{Herencia y genética}

Alrededor del $10 \%$ al $25 \%$ de los bebedores informan no tener resaca, incluso después de consumir grandes cantidades de alcohol, lo que indica que tales individuos toleran bien el alcohol, al menos a corto plazo. ${ }^{22}$ Esto se puede deberse a la variación genética de la ALDH en la población. Faltan datos sobre la proporción de individuos que son especialmente susceptibles a la resaca. Lo más probable es que exista un espectro, desde individuos que nunca experimentan resacas hasta individuos que siempre tienen resacas severas e incapacitantes, después de una noche de consumo excesivo de alcohol. Se desconoce si dicha tolerancia individual al alcohol está asociada con problemas para beber y el riesgo de desarrollar trastornos por consumo de alcohol. Los hijos de alcohólicos han reportado peores síntomas de resaca que otros. ${ }^{42,43}$

\section{Relación con el sexo}

Es importante investigar las diferencias de sexo, ya que su presencia puede tener implicaciones para las consecuencias funcionales de tener resaca. ${ }^{44}$ En un estudio reciente se analizaron datos de 2446 estudiantes (hombres $=50.7 \%$, mujeres $=49.3 \%$ ) los participantes calificaron la presencia y la gravedad de 23 síntomas de resaca. Los datos se agruparon según los rangos estimados de CAS, para garantizar una comparación equitativa entre hombres y mujeres. En el grupo de concentración estimada de alcohol en sangre (CeAS) más bajo $(0 \% \leq$ CeAS $<0.08 \%)$, no se encontraron diferencias significativas de sexo. En el siguiente grupo CeAS $(0.08 \% \leq$ CeAS $<0.11 \%$ ), la gravedad de las náuseas fue significativamente mayor en las mujeres que en los hombres. En el tercer grupo de CeAS $(0.11 \% \leq$ eBAC $<0.2 \%)$, las mujeres reportaron puntajes de severidad más altos en náuseas, cansancio, debilidad y mareos que los hombres, y los hombres informaron la presencia de confusión significativamente más a menudo que las mujeres. En el cuarto grupo de CeAS $(0.2 \% \leq$ CeAS $<0.3 \%$ ), las mujeres reportaron puntajes de severidad más altos en náuseas y cansancio que los hombres. En el grupo más alto de CeAS $(0.3 \% \leq$ CeAS $<0.4 \%)$, no se encontraron diferencias significativas de sexo. Los síntomas con mayores diferencias en las puntuaciones de gravedad fueron de náusea y cansancio $(p=<0.002)$. Aunque las diferencias fueron estadísticamente sig- 
nificativas, fueron de pequeña magnitud $y$, por lo tanto, es probable que sólo tengan poca relevancia clínica. Por lo tanto, los análisis actuales sugieren que los hombres y las mujeres experimentan resacas de maneras comparables. ${ }^{15,44}$

\section{El impacto fisiológico de la resaca de alcohol}

\section{Sueño}

Varios estudios han investigado anteriormente la calidad del sueño con relación a la resaca, ${ }^{38}$ pero la mayoría de estos estudios se basaron en autoinformes y mostraron que el tiempo de sueño a menudo se pierde a expensas del tiempo de bebida. Recientemente se ha demostrado que la calidad del sueño se reduce significativamente después de una noche de consumo excesivo de alcohol. ${ }^{45,46}$ Devenney y cols. ${ }^{47}$ publicaron un estudio utilizando un reloj GENEactiv para monitorear continuamente el sueño y la actividad diurna en una noche para beber y un día de control sin alcohol. Los datos mostraron que la calidad subjetiva del sueño fue significativamente peor después de consumir alcohol que en el día de control $(p=0.046)$, así como significativamente menos reposo $(p=0.001)$ y menos refrescante $(p=0.01)$. El sueño también se consideró más satisfactorio cuando los participantes no consumieron alcohol, aunque sin diferencias significativas $(p=0.07)$.

Estos resultados son corroborados en otros diseños de estudio, en el que los participantes, en la condición de resaca, calificaron su calidad del sueño como significativamente peor $(t)(24)=2.70, p=0.012$, $\mathrm{d}=0.54)$ e informaron significativamente más despertares $(t)=2.47, p=0.021$, $\mathrm{d}=1.80$ ) que en la condición de control. Sin embargo, la duración de los despertares y el tiempo necesario para conciliar el sueño no difieren entre las condiciones. ${ }^{48}$

\section{Rendimiento físico y laboral}

En relación con el rendimiento físico y la RA, se realizó en un grupo de senderismo el efecto de la exposición a alcohol una noche anterior al ejercicio en la caminata de Samaria George, que consta de $15.2 \mathrm{~km}$ a una altitud de $1230 \mathrm{~m}$ sobre el nivel del mar. Los resultados fueron evaluados mediante encuestas sobre sus síntomas antes, durante y después de la caminata. La actividad física fue medida con IPAQ (International Physical Activity Questionnaire) y los síntomas de la resaca fueron medidos con una escala siempre de 11 rubros con un índice de severidad de 0-10, además se evaluó las horas de sueño y la calidad, así como las veces que se levantaron durante la noche para evaluar el descanso. El rendimiento de resistencia física se asoció con experimentar significativamente más agotamiento durante el estado de resaca y los resultados mostraron un aumento del agotamiento en las personas que habían consumido alcohol. ${ }^{12}$

Por otro lado, en una revisión sistemática, se analizó la asociación con el rendimiento laboral, donde se observaron que los niveles más altos de consumo de alcohol se asociaron con niveles más altos de desempeño laboral deteriorado, y estas asociaciones positivas eran considerablemente más propensas que las asociaciones negativas a ser estadísticamente significativas ( $O R=$ 14.00, phi $=0.37, p<0,001)$. Sin embargo, debido a la baja calidad de la investigación y la falta de diseños longitudinales, la evidencia no es concluyente. ${ }^{49}$

\section{Composición del microbioma}

En un estudio naturalista, que consistió en un día de consumo de alcohol y un día de control sin consumo de alcohol, se recogieron muestras de heces y saliva de estos individuos en cada día de prueba y se investigó la composición del microbioma. ${ }^{13}$ El mayor cambio en el microbioma fue el 
aumento de Rothia, que se correlacionó significativamente de manera negativa con la gravedad de la resaca informada. Este hallazgo puede explicarse por la noción de que la presencia de Rothia está asociada con la producción de grandes cantidades de acetaldehído y, por lo tanto, puede influir en la tasa de metabolismo del alcohol. ${ }^{13}$ Esto último podría influir en la presencia y gravedad del siguiente síntoma de resaca de alcohol. ${ }^{38}$

\section{Inmunidad e inflamación}

Se ha sugerido que el estado y el funcionamiento inmunes percibido desempeñan un papel en la patología de la resaca de alcohol. Investigaciones anteriores han encontrado que los bebedores con un estado inmunitario auto informado más pobre son más vulnerables a experimentar resacas por alcohol, ${ }^{11}$ aunque no se ha encontrado una relación directa entre la gravedad de las resacas y el estado inmunitario.

Aurora van de Loo discutió, en la $11^{\circ}$ Reunión del Grupo de Investigación de la Resaca de Alcohol, ${ }^{38}$ los resultados de tres estudios diferentes que evaluaron las concentraciones de citoquinas en la saliva en diferentes momentos después del consumo de alcohol. En el primer estudio controlado, las concentraciones de citoquinas salivales se midieron cada hora durante 8 horas después de que los participantes consumieron alcohol a una CAS del $0.08 \%$. Las citocinas que se pudieron detectar de manera confiable fueron IL-1ß, IL-8, TNF- $\alpha$. En comparación con el día de control sin alcohol, se encontró un aumento significativo de IL-1ß en varios momentos durante el día de la prueba de alcohol. En el segundo estudio las respuestas de las citocinas salivales en el mañana después de una tarde de consumo de alcohol y en la mañana de un día de control se midieron. En el tercer estudio, se siguió el mismo diseño naturalista que en el segundo estudio; sin embargo, las citocinas se evaluaron cada hora durante la resaca y en un día de control. En el segundo estudio, IL-1ß, IL-6, IL-8, TNF- $\alpha$ e IL-10 se pudieron detectar de manera confiable. Se encontraron aumentos significativos asociados con la resaca en las concentraciones de citocinas salivales para IL-6 e IL-10, y el aumento de TNF- $\alpha$ se acercó a su importancia. En el tercer estudio, no se encontraron cambios significativos en las concentraciones de citocinas, pero como se trataba de datos preliminares de sólo nueve sujetos, el bajo tamaño de la muestra probablemente explica la ausencia de cambios estadísticamente significativos. Tomados en conjunto, estos estudios demuestran que el sistema inmunitario responde al consumo excesivo de alcohol. Estos hallazgos sugieren que la respuesta inmune puede estar relacionada con la presencia de resaca de alcohol. Sin embargo, la gravedad de la resaca no se correlacionó significativamente con ninguno de los cambios de citocinas salivales descritos. ${ }^{38}$

\section{El impacto de la resaca en la función mito- condrial}

Recientemente se ha verificado que la resaca es inducida por estrés oxidativo, fenómeno observado en los sinaptosomas de la corteza cerebral y mitocondrias no sinápticas (NS). ${ }^{29,50}$ Los sinaptosomas constituyen un apropiado acercamiento a estudios de bioenergéticos y función mitocondrial en la sinapsis en una variedad de modelos experimentales de las patologías de SNC. Asociada con estrés oxidativo, la acción de las proteínas de desacoplamiento (UCPs) constituye un mecanismo fisiológico por el cual la fuerza motriz de los protones atraviesan la membrana mitocondrial interna disipándola parcialmente, lo que mejora así la carga de estrés oxidativo. ${ }^{31,51,52}$ Se ha observado que el consumo crónico de alcohol induce la expresión de UCP-2 y UCP-4 en los cerebros de ratas. Se ha reportado que la exposición compulsiva altera la regulación de las dinámicas mitocondriales y decrece la producción de ATP 
progresivamente hasta la adultez (en adolescentes). ${ }^{51}$ Hay una elevación significativa de peróxido de hidrógeno y superóxido en sinaptosomas y mitocondrias NS en la RA. También se ha demostrado que la reducción de complejos I y $\mathrm{V}$ inducidos por la exposición de etanol, se acompaña por la disminución del potencial de membrana mitocondrial y de la producción de ATP en el cerebro. Es sabido que los sinaptosomas y mitocondrias no sinápticas presentan una respuesta diferencial específica a las condiciones experimentales como la disfunción mitocondrial inducida por calcio u otros modelos.

Karadayian y cols. ${ }^{52}$ emplearon ratones que recibieron inyecciones (vía peritoneal) de EtOH (3,8 g / kg) o solución salina y se sacrificaron 6 horas después. De la corteza cerebral, fueron aislados mitocondrias NS y sinaptosomas por gradiente de Ficoll. Los resultados mostraron que las mitocondrias NS de animales con RA presentaron una disminución del $26 \%$ en la respiración del estado de malato-glutamato, una reducción del $64 \%$ en el contenido de ATP, ${ }^{3}$ una disminución del $28-37 \%$ en las tasas de producción de ATP (dependiente de glutamato o succinato de malato ${ }^{11}$ respectivamente) y $44 \%$ de inhibición en la actividad del complejo IV. No se observaron cambios en el potencial transmembrana mitocondrial $(\Delta \Psi)$ ni en la expresión de UCP-2 en las mitocondrias NS. La respiración de sinaptosomas condujo la fuga de protones (en presencia de oligomicina) y la capacidad respiratoria de reserva (proporción porcentual entre la respiración máxima y basal) aumentaron $30 \%$ y $15 \%$ en la condición de resaca, respectivamente. El contenido de ATP sinaptosomal disminuyó en un $26 \%$ y las tasas de producción de ATP disminuyeron en un $40-55 \%$ (dependiente de malato-glutamato o succinato, respectivamente) en ratones con RA. Además, se observó una disminución del $24 \%$ en $\Delta \Psi$ y un aumento del $21 \%$ en la expresión de la proteína UCP-2 en sinaptosomas de ra- tones con RA. Además, las actividades de los complejos respiratorios mitocondriales I-III, II-III y IV medidas en sinaptosomas de ratones con RA disminuyeron en un $18 \%$, $34 \%$ y $50 \%$ respectivamente. Los resultados de este estudio revelan que las alteraciones en el estado bioenergético durante la RA podrían deberse principalmente a cambios en la función mitocondrial a nivel de sinapsis. Por lo que la RA provoca un desbalance en la hemostasia redox celular particularmente afectando mitocondrias presentes en sinaptosomas. Además, en la respiración mitocondrial se mostró que, durante la RA, hay una disminución significativa observada en la respiración de malato glutamato en mitocondrias NS.

\section{El impacto cognitivo de las resacas alcohó- licas}

Los efectos del consumo excesivo de alcohol en el funcionamiento cognitivo más allá de la intoxicación a la posterior resaca de alcohol, ha recibido una atención creciente en los últimos años. En una revisión sistemática de 11 artículos reportó que los estudios de alta calidad existentes indicaron una asociación de desarrollar un trastorno por consumo de alcohol cuando se experimentan resacas con frecuencia, ajustadas por la ingesta de alcohol. ${ }^{14}$ Si bien aún no está claro si estas dos consecuencias están relacionadas funcionalmente o cómo están relacionadas, se ha demostrado que los efectos cognitivos agudos de una intoxicación alcohólica en dosis altas se asemejan al patrón de déficit cognitivo observado en pacientes con trastornos por consumo de alcohol, ambos producen alteraciones pronunciadas en el control del funcionamiento cognitivo/ejecutivo, mientras que la automaticidad conductual se conserva comparativamente. ${ }^{53-56}$

Opitz y cols. ${ }^{56}$ indujeron experimentalmente los síntomas de la resaca por una administración estandarizada de bebidas alcohólicas (con alto contenido de congéneres). Los resultados no demostraron 
efectos significativos de la resaca, lo que sugiere que la RA no produjo los mismos déficits funcionales que una intoxicación aguda a altas dosis. Aun así, los análisis bayesianos complementarios revelaron que la resaca perjudica la selección de la respuesta, pero no la inhibición de la respuesta. Este patrón de efectos no puede explicarse con el conocimiento actual sobre cómo el etanol y su metabolito acetaldehído pueden modular la selección de respuesta y la inhibición a través del sistema dopaminérgico o GABAérgico. ${ }^{57,58}$ Sin embargo, esto no ayuda a explicar el hallazgo de una selección ligeramente alterada y una inhibición relativamente conservada. ${ }^{56}$

Devenney y cols. ${ }^{59}$ realizaron un estudio para analizar la atención, el funcionamiento de la memoria y el estado de ánimo en un entorno natural con la RA. Se aplicó un diseño entre sujetos comparando un grupo de resaca con un grupo de control (sin alcohol). Los participantes en el grupo de resaca consumieron una media de 13.8 bebidas alcohólicas la noche antes de la prueba. Las pruebas cognitivas incluyeron la prueba de Stroop, la prueba de Flanker de Eriksen, una prueba de atención dividi$\mathrm{da}$, prueba de cambio de conjunto intradimensional-extradimensional, prueba de memoria de trabajo espacial y prueba de recuperación de palabras libres. La atención selectiva (rendimiento de la prueba Flanker de Stroop y Eriksen) se vio significativamente afectada durante la resaca de alcohol. Sin embargo, el número de errores no difirió significativamente entre los grupos en ninguna tarea. Las evaluaciones del estado de ánimo revelaron que el grupo de resaca se informó niveles significativamente más altos de somnolencia y torpeza en comparación con el grupo de control. Por lo tanto, la atención selectiva se vio significativamente afectada durante la RA. Las diferencias entre la resaca y el grupo de control mostraron significación para otras formas de atención o memoria.

Heffernan y cols. ${ }^{60}$ evaluaron el impacto de la RA en la memoria prospectiva objetiva (MP; memoria para eventos / intenciones futuros). La MP se evaluó utilizando el PRVP, que es una medida de MP basada en una metodología utilizada para estudiar el impacto nocivo del cannabis sobre la MP en adultos y los déficits de MP asociados con el consumo excesivo de alcohol. Un análisis univariado de covarianza (ANCOVA) aplicado a los puntajes de PRVP (controlando por edad, el número de unidades de alcohol consumidas por semana, el número de años que pasaron bebiendo, los puntajes de ansiedad y depresión de HADS) revelaron significativamente menos elementos recordados en el PRVP por el grupo con RA en comparación con el grupo $\sin \mathrm{RA}(\mathrm{F}(1,51)=5.69, \mathrm{p}<0.05 ; 5.16$ vs. 7.51 para AHG y NHG respectivamente). Posteriormente se estudiaron las funciones ejecutivas (FE) aunado a la memoria prospectiva (MP) ya mencionada. Las FE se refiere a un conjunto de procesos cognitivos que facilitan la planificación, la atención, el inicio de acciones apropiadas, la inhibición de estímulos inapropiados, así como la manipulación de información dentro de la memoria de trabajo. Observaron que en el grupo expuesto a resaca los puntajes de FE tanto como la MP se vieron disminuidas, relacionando así que el alcohol disminuye capacidades importantes en las actividades de la vida diaria. ${ }^{8}$ Estos resultados son respaldados por el estudio realizado por Gunn y cols. ${ }^{61}$ en la condición de RA se cometen más errores en las tareas de cambio, en comparación con la condición sin RA. Estos resultados sugieren que una resaca de alcohol perjudica los procesos centrales de la función ejecutiva que son importantes para los comportamientos cotidianos, como la toma de decisiones, la planificación y la flexibilidad mental.

Gunn y cols. observaron que durante la resaca hay deficiencias al día siguiente en memoria a corto plazo, memoria a largo plazo, velocidad psicomotora y atención sostenida, con hallazgos mixtos para los 
efectos del día siguiente en la memoria de trabajo. Estos hallazgos sugieren que las funciones cognitivas específicas pueden verse afectadas la mañana siguiente a una noche de consumo excesivo de alcohol, con implicaciones para el desempeño de la tarea diaria, por ejemplo, conducir. ${ }^{4}$

El Trail-Making-Task- $B$ (TMT-B) mide la atención y la memoria de trabajo con un componente ejecutivo. ${ }^{62}$ Se sabe que la tarea es sensible a las deficiencias cognitivas debidas a enfermedades e intervenciones farmacológicas. Para hacer más accesible esta tarea tradicional de lápiz y papel, se creó y validó una versión en línea, la eTMT-B, para futuros propósitos de investigación. Completaron una serie de medidas, incluida una versión en línea de la escala de gravedad de la resaca de alcohol (AHSS), preguntas sobre cantidad y tipo de bebidas consumidas la noche anterior, y el eTMTB. El tiempo de finalización en el eTMT-B fue significativamente correlacionado con la gravedad de la resaca $(r=0.245, p=$ 0.012 ), la $C A L$ de la noche anterior ( $r=$ 0.197, $p=0.041$ ), y el tiempo dedicado a comer $(r=0.376, p<0.001) .^{3}$ Estos hallazgos confirman que la resaca de alcohol afecta negativamente el funcionamiento cognitivo y que la memoria de trabajo más pobre y el rendimiento ejecutivo se correlacionan con resaca severa. ${ }^{63}$

La resaca de alcohol se caracteriza por una cognición y un estado de ánimo empeorados, las personas con resaca pueden mostrar una respuesta exagerada al estrés. Sin embargo, Benson y cols. ${ }^{48}$ examinaron los efectos de la resaca en el estado de ánimo, la capacidad de realizar múltiples tareas y la reactividad del estrés psicológico a la demanda cognitiva. Utilizaron un marco multitarea de cuatro módulos (MTF, un factor estresante de laboratorio módulos utilizados) y una batería de cuestionarios que evalúan el estado de ánimo, la gravedad de los síntomas de la resaca y el sueño de la noche anterior. La capacidad multitarea se vio potencialmente afectada en la condición de resaca $(p=0.033)$. Hubo efectos principales significativos de la resaca en el estado de alerta ( $p<0.001)$, satisfacción ( $p<0.001)$, ansiedad ( $p=$ $0.003)$ y fatiga mental $(p<0.001)$. Pero al finalizar el MTF no afectó diferencialmente el estado de ánimo. Los puntajes de la NASA-TLX revelaron que participantes calificaron la demanda de carga de trabajo de completar el MTF Púrpura como mentalmente exigente $(p=0.039, d)$ y estresante $(p=0.031)$ durante la condición de resaca. Además, los participantes calificaron su desempeño como peor $(p=0.011)$ y la tarea como más frustrante $(p=0.033)$ en la condición de resaca. A pesar de la conciencia del bajo rendimiento, parece que en el estado de resaca los individuos no pueden recurrir a recursos cognitivos adicionales para compensar y satisfacer las demandas de tareas en curso. Esto tiene claro implicaciones para los comportamientos que requieren complejos multitarea, incluida la conducción. Estos hallazgos confirman los efectos cognitivos y anímicos negativos de la resaca en el estado de ánimo. También demuestran que la resaca se asocia con un mayor esfuerzo percibido durante el desempeño de la tarea.

\section{Investigación de posibles tratamientos}

La mayoría de los tratamientos para la resaca actualmente comercializados carecen de evidencia científica que respalde su eficacia. Para otros compuestos que han sido evaluados por ensayos clínicos, la efectividad normalmente se limita a mitigar un solo síntoma de resaca o ausente por completo. ${ }^{64,65}$ La causa principal de la falta de un tratamiento eficaz para la resaca es el hecho de que se realizan inversiones limitadas en el desarrollo de dichos tratamientos, y que la patología de la resaca de alcohol aún no se ha aclarado por completo. ${ }^{1,66}$ Dos enfoques dominan el desarrollo del tratamiento de la resaca. El primer enfoque apunta a acelerar el metabolismo del alcohol, mientras que el segundo enfo- 
que apunta a moderar la respuesta inmune provocada por el consumo excesivo de alcohol. Para ambos enfoques, existe un respaldo emergente de la evidencia científica. Se ha demostrado que varios compuestos recientemente desarrollados aceleran el metabolismo del alcohol, ${ }^{67}$ reducen los niveles de citoquinas en sangre que aumentaron por el consumo de alcohol.

\section{Productos naturales con propiedades anti-resaca}

Las terapias herbales para la resaca se han utilizado durante varios siglos. Varias plantas y productos naturales mostraron efectos positivos sobre el metabolismo del alcohol en estudios en animales y humanos. Podrían mejorar los niveles de ADH y ALDH en el hígado y disminuir la concen- tración de alcohol en la sangre. Además, que han demostrado ser una protección efectiva contra las lesiones inducidas por el alcohol y una atenuación significativa de los síntomas de la resaca en varios modelos animales y pruebas humanas limitadas. Los mecanismos de acción son principalmente antioxidantes y antiinflamatorios. Los productos naturales han mostrado amplias perspectivas para la prevención y el tratamiento de la resaca y el trastorno por consumo de alcohol (Tabla 2). Sin embargo, es necesario separar e identificar más compuestos bioactivos en las plantas (especialmente plantas medicinales, frutas y verduras), y los mecanismos de acción deben estudiarse más a fondo. ${ }^{68}$ Por lo tanto, aunque prometedor, se necesitan más investigación para su uso.

\section{Tabla 2}

\section{Tratamientos naturales anti-resaca probados en humanos ${ }^{68}$}

\begin{tabular}{|c|c|c|c|c|c|}
\hline $\begin{array}{l}\text { Produc- } \\
\text { tos } \\
\text { naturales }\end{array}$ & $\begin{array}{l}\text { Compo- } \\
\text { nente } \\
\text { activo }\end{array}$ & $\begin{array}{l}\text { Parte de } \\
\text { la planta }\end{array}$ & Sujetos & $\begin{array}{l}\text { Efectos biológicos y mecanismos } \\
\text { de acción }\end{array}$ & $\begin{array}{l}\text { Refe- } \\
\text { rencia }\end{array}$ \\
\hline \multirow[t]{3}{*}{$\begin{array}{l}\text { Pueraria } \\
\text { lobata }\end{array}$} & $\begin{array}{l}\text { Tectori- } \\
\text { dina }\end{array}$ & Flor seca & Humanos & $\begin{array}{l}\text { Reduce los síntomas de la resaca al } \\
\text { promover la eliminación del acetal- } \\
\text { dehído en la sangre }\end{array}$ & (69) \\
\hline & $\begin{array}{l}\text { Puerarin y } \\
\text { daidzein }\end{array}$ & Raíz & Humanos & $\begin{array}{l}\text { Consumo reducido de alcohol en un } \\
\text { entorno naturalista }\end{array}$ & (70) \\
\hline & & & Humanos & $\begin{array}{l}\text { Consumo reducido de alcohol en un } \\
\text { paradigma de borracheras }\end{array}$ & (71) \\
\hline $\begin{array}{l}\text { Pyrus } \\
\text { pyrifolia }\end{array}$ & $\begin{array}{l}\text { Polifeno- } \\
\text { les }\end{array}$ & Frutas & Humanos & $\begin{array}{l}\text { Alivio de la resaca de alcohol a través } \\
\text { de la sangre bajada niveles de alco- } \\
\text { hol y variación genética modificada } \\
\text { de ALDH2 }\end{array}$ & (72) \\
\hline $\begin{array}{l}\text { Zingiber } \\
\text { officinale }\end{array}$ & 6-gingerol & Rizoma & Humanos & $\begin{array}{l}\text { Disminución de los signos y síntomas } \\
\text { de la resaca de alcohol }\end{array}$ & (73) \\
\hline $\begin{array}{l}\text { Opuntia } \\
\text { ficus- } \\
\text { indica }\end{array}$ & $\begin{array}{l}\text { Ginsenó- } \\
\text { sidos }\end{array}$ & $\begin{array}{l}\text { Raíz de } \\
\text { ginsenósi- } \\
\text { dos }\end{array}$ & Humanos & $\begin{array}{l}\text { Alivio de los síntomas de la resaca a } \\
\text { través de la reducción de la espira- } \\
\text { ción y niveles de alcohol en plasma y } \\
\text { gravedad de la resaca }\end{array}$ & (74) \\
\hline
\end{tabular}


Ventajas y limitaciones de los diseños de estudios naturalistas y su implementación en la investigación de la resaca de alcohol

En la investigación de la resaca de alcohol, tanto el diseño naturalista como el ensayo controlado aleatorizado (ECA) se emplean con éxito para estudiar las causas, las consecuencias y los tratamientos de la resaca. Aunque se aplica cada vez más en las ciencias sociales y la investigación médica, la idoneidad de los diseños de estudios naturalistas sigue siendo un tema de debate. En ambos tipos de diseño de estudio, la evaluación de los participantes y la realización de evaluaciones en el sitio (por ejemplo, pruebas psicométricas, cuestionarios y evaluaciones de biomarcadores) suelen ser igualmente rigurosos y siguen los mismos procedimientos operativos estándar. Sin embargo, difieren en los niveles de monitoreo y restricciones impuestas sobre el comportamiento de los participantes antes de que se realicen las evaluaciones (por ejemplo, comportamientos de consumo de alcohol que resultan en la resaca del día siguiente). Estos comportamientos están altamente controlados en un ECA y no controlados en estudios naturalistas. Como resultado, la mayor diferencia entre los estudios naturalistas y los ECAs es su validez ecológica, que generalmente es significativamente menor para los ECAs y el grado de estandarización de la intervención experimental, que generalmente es significa-

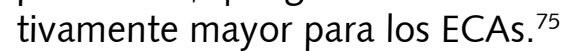

El diseño del ECA se caracteriza por tener varios criterios de inclusión, exclusión e interrupción que se aplican a los participan- tes, incluidas las normas de estilo de vida con respecto, por ejemplo, al consumo de alcohol, drogas y al tabaquismo. Los ECA son idealmente doble o triple ciego para evitar influir en el resultado del estudio, y los participantes se asignan aleatoriamente a las condiciones de tratamiento. El orden de tratamiento es variado (cruzado) para tener en cuenta los efectos de aprendizaje u orden. Sin embargo, este nivel de control tiene el costo de los ECA que crean situaciones altamente artificiales, que carecen de validez ecológica y que difieren potencialmente de los efectos observados en la vida cotidiana de los participantes. Por otro lado, el objetivo del diseño del estudio naturalista es imitar la vida real tan de cerca como sea posible, y como tal se caracteriza por un mínimo de reglas de estilo de vida para los participantes, en el que los investigadores no interfieren (activamente) con sus actividades. Por lo tanto, varios comportamientos y actividades de los participantes no están estandarizados ni regulados por un protocolo de estudio. Los participantes continúan su vida normal y pueden visitar el sitio de prueba para realizar evaluaciones o recolectar muestras biológicas o incluso pueden realizar estas evaluaciones mientras permanecen en su entorno habitual. El diseño naturalista se utiliza cada vez más en diversas áreas de investigación y se ha aplicado con éxito en estudios de fase III e investigación de farmacovigilancia. ${ }^{75}$ En la Tabla 3 se resumen las principales características entre ECA y los diseños de estudio naturalistas.

\title{
Tabla 3
}

\begin{abstract}
Características comunes y diferencias entre los ensayos controlados aleatorios (ECA) y los diseños de estudios naturalistas ${ }^{75}$
\end{abstract}

\begin{tabular}{|l|l|l|}
\hline DISEÑO DE ESTUDIO & $\begin{array}{l}\text { ESTUDIO CONTROLADO } \\
\text { ALEATORIZADO }\end{array}$ & $\begin{array}{l}\text { DISEÑO DE ESTUDIO } \\
\text { NATURALISTA }\end{array}$ \\
\hline Validez ecológica & Medio-bajo & Alto \\
\hline
\end{tabular}




\begin{tabular}{|c|c|c|}
\hline Validez externa & Medio-bajo & Alto \\
\hline Validez interna & Alto & Bajo-medio \\
\hline Validez de criterio & Alto & Alto \\
\hline Validez de constructo & Alto & Alto \\
\hline TAMIZAJE & Controlado, por protocolo & Controlado, por protocolo \\
\hline $\begin{array}{l}\text { Criterios de inclusión y exclu- } \\
\text { sión }\end{array}$ & $\mathrm{Si}$ & $\mathrm{Si}$ \\
\hline $\begin{array}{l}\text { Familiarización con los procedi- } \\
\text { mientos y las pruebas }\end{array}$ & $\mathrm{Si}$ & $\mathrm{Si}$ \\
\hline SESIONES DE BEBIDA & Por control, supervisadas & Sin control, no supervisadas \\
\hline Instrucciones & Por control & No o mínimas \\
\hline Cantidad y tipo de bebida & Preestablecida, estandarizada & Autoinducidas \\
\hline $\begin{array}{l}\text { Inicio y final del tiempo para } \\
\text { beber }\end{array}$ & Preestablecida, estandarizada & Autoinducidas \\
\hline $\begin{array}{l}\text { Comportamientos durante la } \\
\text { bebida }\end{array}$ & Restringida, por protocolo & Libre \\
\hline Aspectos sociales de la bebida & Con extraños & Libre \\
\hline Evaluaciones en tiempo real & Sí & $\begin{array}{l}\text { Posiblemente vía tecnología } \\
\text { móvil }\end{array}$ \\
\hline Ingesta de bebida y agua & Restringida, por protocolo & Libre \\
\hline Tabaquismo & Restringido por protocolo & Libre \\
\hline SUEÑO & Controlado y supervisado & Sin control y no supervisado \\
\hline Hora de ir a dormir y despertar & Restringido, por protocolo & \\
\hline $\begin{array}{l}\text { Higiene del sueño y comporta- } \\
\text { mientos asociados }\end{array}$ & Restringido, por protocolo & \\
\hline Monitoreo en tiempo real & Sí & $\begin{array}{l}\text { Posiblemente vía tecnología } \\
\text { móvil }\end{array}$ \\
\hline Ambiente de sueño & Unidas de sueño & En casa u otro lugar \\
\hline DÍAS DE PRUEBA & Controlado y estandarizado & Controlado y estandarizado \\
\hline $\begin{array}{l}\text { Pruebas psicométricas, evalua- } \\
\text { ción del estado de animo }\end{array}$ & Estandarizado y validado & Estandarizado y validado \\
\hline Tiempo de pruebas & $\begin{array}{l}\text { Tiempo por protocolo y preesta- } \\
\text { blecido }\end{array}$ & $\begin{array}{l}\text { Tiempo por protocolo y prees- } \\
\text { tablecido }\end{array}$ \\
\hline $\begin{array}{l}\text { Conductancia de los procedi- } \\
\text { mientos de estudio }\end{array}$ & Por protocolo & Por protocolo \\
\hline Supervisión, monitoreo & Sí & Sí \\
\hline
\end{tabular}




\section{Discusión}

Críticamente, todos los modelos de resaca deben basarse en la definición actual de resaca de alcohol, que se describe anteriormente. ${ }^{38}$ En primer lugar, es importante determinar el inicio de la resaca dentro del modelo animal. Esto se logra midiendo la concentración de etanol dentro de la sangre, con el inicio de la resaca una vez que la concentración de etanol en la sangre vuelve a cero. ${ }^{16,52}$ Una segunda consideración crítica para el desarrollo de un modelo animal es la compleja sintomatología experimentada en una resaca: más de 47 síntomas, incluidos los síntomas físicos y psicológicos, pueden modelarse mejor en animales completando tareas de comportamiento múltiples y variadas que cubren un gama de síntomas. ${ }^{3} \mathrm{Al}$ considerar qué pruebas de comportamiento incluir en un modelo de resaca de alcohol, es importante tener en cuenta la amplia variedad sintomática que acompaña a la resaca de alcohol. Es de destacar que incluso hay una amplia variedad de síntomas interindividuales dependiendo de la aparición específica de la resaca. Por lo tanto, en contraste con la investigación en humanos, un criterio de valoración primario (gravedad de la resaca general informada) no es posible en la investigación con animales, pero es una combinación de resultados de varias pruebas de comportamiento. ${ }^{38}$ Finalmente, tanto la dosis como la vía de administración de etanol deben considerarse cuidadosamente. La experiencia humana de la resaca de alcohol sigue a una sola dosis de consumo excesivo de alcohol en la que normalmente se experimentan altos niveles de intoxicación. La literatura ha encontrado que entre 3 y $6 \mathrm{~g} / \mathrm{kg}$ han inducido con éxito la intoxicación en ratones y ratas, lo que puede confirmarse por la pérdida del reflejo de enderezamiento. ${ }^{76}$ La ruta intragástrica imita el consumo excesivo de alcohol en humanos $y$, por lo tanto, es la mejor ruta para usar en un modelo animal de resaca de alcohol. ${ }^{38}$
Las herramientas de medición precisas son esenciales para evaluar la gravedad de la resaca, por ejemplo, en estudios experimentales y naturalistas, en estudios de intervención que examinan la eficacia del tratamiento y en investigaciones de encuestas. Por ello se analizaron las distintas escalas que hay para medir los síntomas presentados en la resaca comparando 3 escalas habituales contra una escala de 1 rubro, concluyendo que las escalas habituales con muchos puntos relacionados con los síntomas suelen sobrestimar los síntomas de la resaca, dando como resultado generación de sesgo en la medición de síntomas y por lo tanto al evaluar un efecto terapéutico. ${ }^{24}$

Otro reto importante en la investigación de la RA es el diseño de estudio idóneo. Es difícil poder realizar un análisis sistemático de los estudios más actuales debido a la dificultad de comparar dos diseños de estudio diferente (ECA y diseño de estudio naturalista). ${ }^{75} \mathrm{El}$ diseño de estudio naturalista ha tomado mucha popularidad en su aplicación; sin embargo, las evaluaciones a menudo se limitan a ser retrospectivas y subjetivas a través de autoinformes. Cuando se basa completamente en autoinformes, el sesgo de memoria y la pérdida de memoria pueden tener un impacto significativo en la precisión de los datos recopilados. ${ }^{48} \mathrm{Si}$ se llega a un consenso sobre el diseño ideal para futuras investigaciones, permitiría hacer revisiones sistemáticas y metaanálisis de los datos generados. Además de que en futuras investigaciones se estudien a más sujetos dentro de cada estudio para que los resultados sean más significativos.

Es importante que, de igual forma, se evalúe también los síntomas individuales de la resaca. Esto se puede hacer usando una de las escalas de resaca existentes, o simplemente evaluando los síntomas individuales de interés a través de puntajes de gravedad de 1 elemento específico de síntomas. Sin embargo, el juicio de la eficacia 
ca con un día sin resaca para capturar el efecto de resaca "verdadero". Este último no se aplica a los estudios de intervención, donde se debe hacer una comparación directa de las puntuaciones de los síntomas entre el tratamiento y el placebo para evaluar una posible diferencia entre las dos condiciones de resaca.

\section{Conclusiones}

Esperamos que posteriormente se indague sobre el impacto de la RA a través de variables como grupos étnicos y con estudios genéticos que aporten datos más completos para un análisis profundo de la información. Es importante ampliar nuestros conocimientos sobre RA. Esto nos permitirá contar con más elementos, evidencia y herramientas sobre los efectos adversos del consumo de alcohol agudo y crónico, formando parte del nivel preventivo con el paciente y la comunidad.

\section{Bibliografía}

1. Verster JC, Scholey A, van de Loo AJAE, Benson $\mathrm{S}$, Stock A-K. Updating the Definition of the Alcohol Hangover. J Clin Med [Internet]. 2020 Mar 18;9(3):823. Available from: https://www.mdpi. com/2077-0383/9/3/823

2. Vatsalya $V$, Stangl BL, Schmidt VY, Ramchandani VA. Characterization of hangover following intravenous alcohol exposure in social drinkers: methodological and clinical implications. Addict Biol [Internet]. 2018 Jan;23(1):493-502. Available from: http:// doi.wiley.com/10.1111/adb.12469

3. Royle S, Owen L, Roberts D, Marrow L. Pain Catastrophising Predicts Alcohol Hangover Severity and Symptoms. J Clin Med [Internet]. 2020 Jan 20;9(1):280. Available from: https://www.mdpi. com/2077-0383/9/1/280

4. Gunn $C$, Mackus $M$, Griffin $C$, Munafò $M R$, Adams S. A systematic review of the next-day effects of heavy alcohol consumption on cognitive performance. Addiction [Internet]. 2018 Dec;113(12):218293. Available from: http://dx.doi.org/10.1111/ add. 14404

5. Hartung B, Schwender $H$, Mindiashvili N, Ritz-Timme S, Malczyk A, Daldrup T. The effect of alcohol hangover on the ability to ride a bicycle. Int J Legal Med [Internet]. 2015;129(4):751-8. Available from: https://doi.org/10.1007/s00414-015-1194-2

6. Gjerde H, Christophersen AS, Moan IS, Yttredal B, Walsh JM, Normann PT, et al. Use of alcohol and drugs by Norwegian employees: A pilot study using questionnaires and analysis of oral fluid. J Occup
Med Toxicol. 2010;5(1):1-8.

7. Vatsalya $V$, Hassan $H Z$, Kong $M$, Stangl $B L$, Schwandt $M L$, Schmidt-Teron VY, et al. Exacerbation of Hangover Symptomology Significantly Corresponds with Heavy and Chronic Alcohol Drinking: A Pilot Study. J Clin Med [Internet]. 2019 Nov 12;8(11):1943. Available from: https://www.mdpi.com/20770383/8/11/1943

8. Heffernan T, Samuels A, Hamilton C, McGrathBrookes M. Alcohol Hangover Has Detrimental Impact Upon Both Executive Function and Prospective Memory. Front Psychiatry [Internet]. 2019 May 17;10(MAY):1-6. Available from: https://www. frontiersin.org/article/10.3389/fpsyt.2019.00282/ full

9. Hagström $H$, Hemmingsson $T$, Discacciati $A$, Andreasson A. Risk Behaviors Associated with Alcohol Consumption Predict Future Severe Liver Disease. Dig Dis Sci [Internet]. 2019 Jul 14;64(7):2014-23. Available from: http://dx.doi.org/10.1007/s10620019-05509-6

10. Raasveld SJ, Hogewoning A, Van de Loo AJAE, De Zeeuw R, Bosma ER, Bouwmeester $\mathrm{NH}$, et al. P.1.f.006 Cytokine concentrations after heavy alcohol consumption in people with and without a hangover. Eur Neuropsychopharmacol [Internet]. 2015 Sep;25:S228. Available from: http://www.sciencedirect.com/science/article/pii/S0924977X15302455

11. van de Loo A, Mackus $M$, van Schrojenstein Lantman $M$, Kraneveld A, Brookhuis K, Garssen J, et al. Susceptibility to Alcohol Hangovers: The Association with Self-Reported Immune Status. Int J Environ Res Public Health [Internet]. 2018 Jun 18;15(6):1286. 
Available from: http://dx.doi.org/10.3390/ijerph15061286

12. Verster JC, Anogeianaki A, Kruisselbrink D, Alford C, Stock A-K. Relationship between Alcohol Hangover and Physical Endurance Performance: Walking the Samaria Gorge. J Clin Med [Internet]. 2019 Dec 31;9(1):114. Available from: https://www.mdpi. com/2077-0383/9/1/114

13. Moritani K, Takeshita T, Shibata $Y$, Ninomiya T, Kiyohara $Y$, Yamashita $Y$. Acetaldehyde production by major oral microbes. Oral Dis. 2015;21(6):748-54.

14. ML M, AL S, JS T. Alcohol Hangover and Risk of Drinking Problems and Alcohol Use Disorder: A Systematic Review. J Alcohol Drug Depend [Internet]. 2017;05(01):1-7. Available from: https://www. esciencecentral.org/journals/alcohol-hangoverand-risk-of-drinking-problems-and-alcohol-usedisorder-a-systematic-review-2329-6488-1000255. php?aid $=85801$

15. Alford C, Martinkova Z, Tiplady B, Reece R, Verster JC. The Effects of Alcohol Hangover on Mood and Performance Assessed at Home. J Clin Med [Internet]. 2020 Apr 9;9(4):1068. Available from: https:// www.mdpi.com/2077-0383/9/4/1068

16. van Schrojenstein Lantman $M$, van de Loo $A$, Mackus $M$, Verster J. Development of a Definition for the Alcohol Hangover: Consumer Descriptions and Expert Consensus. Curr Drug Abuse Rev [Internet]. 2017 May 17;9(2):148-54. Available from: http:// www.eurekaselect.com/node/150158/article

17. Thompson C, Milton S, Egan M, Lock K. Down the local: A qualitative case study of daytime drinking spaces in the London Borough of Islington. Int J Drug Policy [Internet]. 2018;52:1-8. Available from: http://10.0.3.248/j.drugpo.2017.11.019

18. Ally AK, Lovatt $M$, Meier PS, Brennan A, Holmes J. Developing a social practice-based typology of British drinking culture in 2009-2011: implications for alcohol policy analysis. Addiction [Internet]. 2016 Sep;111(9):1568-79. Available from: http://10.0.4.87/add.13397

19. Penning R, McKinney A, Verster JC. Alcohol hangover symptoms and their contribution to the overall hangover severity. Alcohol Alcohol. 2012;47(3):248-52.

20. Rohsenow DJ, Howland J, Minsky SJ, Greece J, Almeida A, Roehrs TA. The Acute Hangover Scale: A new measure of immediate hangover symptoms. Addict Behav [Internet]. 2007 Jun;32(6):1314-20. Available from: https://linkinghub.elsevier.com/retrieve/pii/S0022202X15370834

21. Slutske WS, Piasecki TM, Hunt-Carter EE. Development and Initial Validation of the Hangover Symptoms Scale: Prevalence and Correlates of Hangover Symptoms in College Students. Alcohol Clin Exp Res [Internet]. 2003 Sep;27(9):1442-50. Available from: http://doi.wiley.com/10.1097/01. ALC.0000085585.81711.AE

22. Penning R, McKinney A, Bus LD, Olivier B, Slot $K$, Verster JC. Measurement of alcohol hangover severity: development of the Alcohol Hangover Severity Scale (AHSS). Psychopharmacology (Berl) [Internet]. 2013 Feb 25;225(4):803-10. Available from: http:// link.springer.com/10.1007/s00213-012-2866-y

23. Hogewoning $A$, van de Loo $A$, Mackus $M$, Raasveld $S$, De Zeeuw $R$, Bosma $E$, et al. Characteristics of social drinkers with and without a hangover after heavy alcohol consumption. Subst Abuse Rehabil [Internet]. 2016 Nov; Volume 7:161-7. Available from: https://www.dovepress.com/characteristicsof-social-drinkers-with-and-without-a-hangoverafter-h-peer-reviewed-article-SAR

24. Verster JC, van de Loo AJAE, Benson S, Scholey A, Stock A-K. The Assessment of Overall Hangover Severity. J Clin Med [Internet]. 2020 Mar 13;9(3):786. Available from: https://www.mdpi.com/20770383/9/3/786

25. Verster JC, van Schrojenstein Lantman $M$, Mackus $M$, van de Loo AJAE, Garssen J, Scholey A. Differences in the Temporal Typology of Alcohol Hangover. Alcohol Clin Exp Res. 2018;42(4):691-7.

26. Dueland AN. Headache and Alcohol. Headache J Head Face Pain [Internet]. 2015 Jul;55(7):1045-9. Available from: http://doi.wiley.com/10.1111/ head.12621

27. Verster, Vermeulen, Loo, Balikji, Kraneveld, Garssen, et al. Dietary Nutrient Intake, Alcohol Metabolism, and Hangover Severity. J Clin Med [Internet]. 2019 Aug 27;8(9):1316. Available from: https://www. mdpi.com/2077-0383/8/9/1316

28. Heier $\mathrm{C}$, Xie H, Zimmermann R. Nonoxidative ethanol metabolism in humans-from biomarkers to bioactive lipids. IUBMB Life [Internet]. 2016 Dec;68(12):91623. Available from: http://doi.wiley.com/10.1002/ iub.1569

29. Karadayian AG, Malanga G, Czerniczyniec A, Lombardi $\mathrm{P}$, Bustamante J, Lores-Arnaiz $\mathrm{S}$. Free radical production and antioxidant status in brain cortex non-synaptic mitochondria and synaptosomes at alcohol hangover onset. Free Radic Biol Med [Internet]. 2017 Jul;108:692-703. Available from: https://linkinghub.elsevier.com/retrieve/pii/ S0891584917305476

30. Mackus $M$, Van de Loo AJAE, Korte-Bouws GAH, Van Neer RHP, Wang $X$, Nguyen TT, et al. Urine methanol concentration and alcohol hangover severity. Alcohol [Internet]. 2017 Mar;59:37-41. Available from: https://linkinghub.elsevier.com/retrieve/ pii/S0741832916301537

31. Palmer $E$, Tyacke $R$, Sastre $M$, Lingford-Hughes $A$, Nutt D, Ward RJ. Alcohol Hangover: Underlying Biochemical, Inflammatory and Neurochemical Mechanisms. Alcohol Alcohol [Internet]. 2019 May 1;54(3):196-203. Available from: https://academic. oup.com/alcalc/article/54/3/196/5420612

32. Van de Loo A, Mackus M, Korte-Bouws G, Brookhuis K, Garssen J, Verster J. Urine ethanol concentration and alcohol hangover severity. Psychopharmacology (Berl) [Internet]. 2017 Jan 28;234(1):73-7. Available from: http://10.0.3.239/s00213-016-4437-0

33. Gandy JJ, Laurens I, Snyman JR. Potentiated clinoptilolite reduces signs and symptoms associated with veisalgia. Clin Exp Gastroenterol. 2015;8:271-7.

34. Verster JC, Kruisselbrink LD, Slot KA, Anogeianaki A, Adams $S$, Alford $C$, et al. Sensitivity to Experiencing 
Alcohol Hangovers: Reconsideration of the $0.11 \%$ Blood Alcohol Concentration (BAC) Threshold for Having a Hangover. J Clin Med [Internet]. 2020 Jan 9;9(1):179. Available from: https://www.mdpi. com/2077-0383/9/1/179

35. Hammer JH, Parent MC, Spiker DA. Mental Help Seeking Attitudes Scale (MHSAS): Development, reliability, validity, and comparison with the ATSPPHSF and IASMHS-PO. J Couns Psychol [Internet]. 2018 Jan;65(1):74-85. Available from: http://www. who.int/substance abuse/publications/global_alcohol_report/msbgsruprofiles.pdf\% 0Ahttp://wwww. ncbi.nlm.nih.gov/pubmed/29355346

36. Islam T, Wei J, Sheikh Z, Hameed Z, Azam RI. Determinants of compulsive buying behavior among young adults: The mediating role of materialism. J Adolesc [Internet]. 2017 Dec;61:117-30. Available from: https://linkinghub.elsevier.com/retrieve/pii/ S0140197117301586

37. Kruisselbrink LD, Bervoets AC, de Klerk S, van de Loo AJAE, Verster JC. Hangover resistance in a Canadian University student population. Addict Behav Reports [Internet]. 2017 Jun;5:14-8. Available from: http:// dx.doi.org/10.1016/j.abrep.2017.01.001

38. Palmer EOC, Arnoldy L, Ayre E, Benson S, Balikji S, Bruce $G$, et al. Proceedings of the 11th Alcohol Hangover Research Group Meeting, in Nadi, Fiji. Proceedings [Internet]. 2020 Feb 18;43(1):1. Available from: https://www.mdpi.com/2504-3900/43/1/1

39. Mackus $M$, van Schrojenstein Lantman $M$, Van de Loo AJAE, Kraneveld AD, Garssen J, Brookhuis KA, et al. Alcohol metabolism in hangover sensitive versus hangover resistant social drinkers. Drug Alcohol Depend [Internet]. 2018;185(November 2017):351-5. Available from: https://doi.org/10.1016/j.drugalcdep.2017.11.040

40. Roohani N, Hurrell R, Kelishadi R, Schulin R. Zinc and its importance for human health: An integrative review. J Res Med Sci Off J Isfahan Univ Med Sci. 2013 Feb;18(2):144-57.

41. Kirkland JB, Meyer-Ficca ML. Niacin. In: Advances in food and nutrition research [Internet]. United States 2018. p. 83-149. Available from: https://linkinghub. elsevier.com/retrieve/pii/S1043452617300396

42. Verster J. Editorial: [The "Hair of the Dog": A Useful Hangover Remedy or a Predictor of Future Problem Drinking?]. Curr Drug Abus Rev [Internet]. 2009 Jan 1;2(1):1-4. Available from: http://www.eurekaselect.com/openurl/content.php? genre $=$ article \&issn $=1874$ 4737 \& volume $=2$ \&issue $=1 \&$ spage $=1$

43. Stephens R, Holloway K, Grange JA, Owen L, Jones K, Kruisselbrink D. Does familial risk for alcohol use disorder predict alcohol hangover? Psychopharmacology (Berl) [Internet]. 2017 Jun 16;234(12):1795802. Available from: https://doi.org/10.1007/ s00213-017-4585-x

44. van Lawick van Pabst, Devenney, Verster. Sex Differences in the Presence and Severity of Alcohol Hangover Symptoms. J Clin Med. 2019;8(6):867.

45. van Schrojenstein Lantman $M$, Mackus $M$, van de Loo AJAE, Verster JC. The impact of alcohol hango- ver symptoms on cognitive and physical functioning, and mood. Hum Psychopharmacol Clin Exp [Internet]. 2017 Sep;32(5):e2623. Available from: http:// doi.wiley.com/10.1002/hup.2623

46. Devenney LE, Coyle KB, Roth T, Verster JC. Sleep after Heavy Alcohol Consumption and Physical Activity Levels during Alcohol Hangover. J Clin Med [Internet]. 2019 May 27;8(5):752. Available from: https://pubmed.ncbi.nlm.nih.gov/31137775

47. Devenney LE, Coyle KB, Roth T, Verster JC. Sleep after Heavy Alcohol Consumption and Physical Activity Levels during Alcohol Hangover. J Clin Med [Internet]. 2019 May 27;8(5):752. Available from: http:// dx.doi.org/10.3390/jcm8050752

48. Benson S, Ayre E, Garrisson H, Wetherell MA, Verster JC, Scholey A. Alcohol Hangover and Multitasking: Effects on Mood, Cognitive Performance, Stress Reactivity, and Perceived Effort. J Clin Med [Internet]. 2020 Apr 17;9(4):1154. Available from: https://www.mdpi.com/2077-0383/9/4/1154

49. Thørrisen MM, Bonsaksen $T$, Hashemi N, Kjeken I, van Mechelen W, Aas RW. Association between alcohol consumption and impaired work performance (presenteeism): a systematic review. BMJ Open [Internet]. 2019 Jul 16;9(7):e029184. Available from: http://bmjopen.bmj.com/lookup/doi/10.1136/ bmjopen-2019-029184

50. Karadayian AG, Bustamante J, Czerniczyniec A, Lombardi P, Cutrera RA, Lores-Arnaiz S. Alcohol hangover induces mitochondrial dysfunction and free radical production in mouse cerebellum. Neuroscience [Internet]. 2015 Sep;304:47-59. Available from: https://linkinghub.elsevier.com/retrieve/pii/ S0306452215006193

51. Tapia-Rojas C, Carvajal FJ, Mira RG, Arce C, LermaCabrera JM, Orellana JA, et al. Adolescent Binge Alcohol Exposure Affects the Brain Function Through Mitochondrial Impairment. Mol Neurobiol. 2018 May;55(5):4473-91.

52. Karadayian AG, Lombardi P, Bustamante J, LoresArnaiz $\mathrm{S}$. Alcohol hangover effects on brain cortex non-synaptic mitochondria and synaptosomes bioenergetics. Alcohol [Internet]. 2019 Jun;77:11323. Available from: http://dx.doi.org/10.1016/j.alcohol.2018.10.010

53. Bjork JM, Gilman JM. The effects of acute alcohol administration on the human brain: Insights from neuroimaging. Neuropharmacology [Internet]. 2014 Sep;84:101-10. Available from: https://linkinghub. elsevier.com/retrieve/pii/S0028390813003572

54. Field M, Schoenmakers T, Wiers R. Cognitive Processes in Alcohol Binges: A Review and Research Agenda. Curr Drug Abus Rev [Internet]. 2008 Nov 1;1(3):26379. Available from: http://www.eurekaselect.com/ openurl/content. php?genre $=$ article \&issn $=1874$ 4737\& volume $=1$ \&issue $=3 \&$ spage $=263$

55. Chmielewski WX, Zink N, Chmielewski KY, Beste C, Stock A-K. How high?dose alcohol intoxication affects the interplay of automatic and controlled processes. Addict Biol [Internet]. 2020 Jan 18;25(1):e12700. Available from: https://onlinelibrary.wiley.com/doi/abs/10.1111/adb.12700 
56. Opitz A, Hubert J, Beste C, Stock A-K. Alcohol Hangover Slightly Impairs Response Selection but not Response Inhibition. J Clin Med [Internet]. 2019 Aug 27;8(9):1317. Available from: https://www.mdpi. com/2077-0383/8/9/1317

57. Quetscher C, Yildiz A, Dharmadhikari S, Glaubitz B, Schmidt-Wilcke T, Dydak U, et al. Striatal GABA-MRS predicts response inhibition performance and its cortical electrophysiological correlates. Brain Struct Funct [Internet]. 2014/08/26. $2015 \mathrm{Nov}$ 26;220(6):3555-64. Available from: https://pubmed.ncbi.nlm.nih.gov/25156575

58. Ramdani C, Carbonnell L, Vidal F, Béranger C, Dagher A, Hasbroucq T. Dopamine precursors depletion impairs impulse control in healthy volunteers. Psychopharmacology (Berl) [Internet]. 2015 Jan 20;232(2):477-87. Available from: http://link.springer.com/10.1007/s00213-014-3686-z

59. Devenney LE, Coyle KB, Verster JC. Memory and attention during an alcohol hangover. Hum Psychopharmacol. 2019;34(4):1-7.

60. Heffernan T. A State of Alcohol Hangover Impedes Everyday Prospective Memory. Front Hum Neurosci [Internet]. 2018 Sep 19;12:348. Available from: http://dx.doi.org/10.3389/fnhum.2018.00348

61. Gunn C, Fairchild G, Verster JC, Adams S. The Effects of Alcohol Hangover on Executive Functions. J Clin Med [Internet]. 2020 Apr 17;9(4):1148. Available from: https://www.mdpi.com/20770383/9/4/1148

62. Fellows RP, Dahmen J, Cook D, Schmitter-Edgecombe M. Multicomponent analysis of a digital Trail Making Test. Clin Neuropsychol [Internet]. 2016/10/03. 2017 Jan;31(1):154-67. Available from: https:// pubmed.ncbi.nlm.nih.gov/27690752

63. Scholey A, Benson S, Kaufman J, Terpstra C, Ayre $E$, Verster J, et al. Effects of Alcohol Hangover on Cognitive Performance: Findings from a Field/Internet Mixed Methodology Study. J Clin Med [Internet]. 2019 Mar 30;8(4):440. Available from: http:// dx.doi.org/10.3390/jcm8040440

64. Mackus $M$, Lantman $M$ van $S$, JAE van de Loo A, Nutt D, Verster JC. An effective hangover treatment: Friend or foe? Drug Sci Policy Law [Internet]. 2017 Jan 17;3:205032451774103. Available from: http://journals.sagepub.com/ doi/10.1177/2050324517741038

65. Jayawardena R, Thejani $T$, Ranasinghe $P$, Fernando D, Verster JC. Interventions for treatment and/or prevention of alcohol hangover: Systematic review. Hum Psychopharmacol Clin Exp [Internet]. 2017 Sep;32(5):e2600. Available from: http://doi.wiley. com/10.1002/hup. 2600

66. Tipple CT, Benson S, Scholey A. A Review of the Physiological Factors Associated with Alcohol Hangover. Curr Drug Abuse Rev [Internet]. 2017 May 17;9(2):93-8. Available from: http://www.eurekaselect.com/node/149869/article

67. Lee $M-H$, Kwak JH, Jeon $G$, Lee J-W, Seo J-H, Lee $\mathrm{H}-\mathrm{S}$, et al. Red ginseng relieves the effects of alcohol consumption and hangover symptoms in healthy men: a randomized crossover study. Food Funct
[Internet]. 2014;5(3):528. Available from: http:// dx.doi.org/10.1039/C3FO60481K

68. Wang F, Li Y, Zhang Y-J, Zhou Y, Li S, Li H-B. Natural Products for the Prevention and Treatment of Hangover and Alcohol Use Disorder. Molecules [Internet]. 2016 Jan 7;21(1):64. Available from: http:// www.mdpi.com/1420-3049/21/1/64

69. Yamazaki T, Hosono T, Matsushita Y, Kawashima K, Someya $M$, Nakajima $Y$, et al. Pharmacological studies on Puerariae Flos. IV: Effects of Pueraria thomsonii dried flower extracts on blood ethanol and acetaldehyde levels in humans. Int J Clin Pharmacol Res. 2002;22(1):23-8.

70. Lukas SE, Penetar D, Berko J, Vicens L, Palmer C, Mallya $G$, et al. An Extract of the Chinese Herbal Root Kudzu Reduces Alcohol Drinking by Heavy Drinkers in a Naturalistic Setting. Alcohol Clin Exp Res [Internet]. 2005 May;29(5):756-62. Available from: http://doi.wiley.com/10.1097/01. ALC.0000163499.64347.92

71. Penetar DM, Toto LH, Lee DY-W, Lukas SE. A single dose of kudzu extract reduces alcohol consumption in a binge drinking paradigm. Drug Alcohol Depend [Internet]. 2015/05/27. 2015 Aug 1;153:194-200. Available from: https://pubmed.ncbi.nlm.nih. gov/26048637

72. Lee H-S, Isse T, Kawamoto T, Baik HW, Park JY, Yang $M$. Effect of Korean pear (Pyruspyrifolia cV. Shingo) juice on hangover severity following alcohol consumption. Food Chem Toxicol [Internet]. 2013 Aug;58:101-6. Available from: https://linkinghub. elsevier.com/retrieve/pii/S0278691513002342

73. Takahashi M, Li W, Koike K, Sadamoto K. Clinical effectiveness of KSS formula, a traditional folk remedy for alcohol hangover symptoms. J Nat Med [Internet]. 2010 Oct 18;64(4):487-91. Available from: http://link.springer.com/10.1007/s11418010-0430-9

74. Wiese J, McPherson S, Odden MC, Shlipak MG. Effect of Opuntia ficus indica on Symptoms of the Alcohol Hangover. Arch Intern Med [Internet]. 2004 Jun 28;164(12):1334. Available from: http://archinte. jamanetwork.com/article.aspx?doi=10.1001/archinte.164.12.1334

75. Verster JC, van de Loo AJAE, Adams S, Stock A-K, Benson S, Scholey A, et al. Advantages and Limitations of Naturalistic Study Designs and Their Implementation in Alcohol Hangover Research. J Clin Med [Internet]. 2019 Dec 6;8(12):2160. Available from: https://www.mdpi.com/2077-0383/8/12/2160

76. Walter TJ, Crews FT. Microglial depletion alters the brain neuroimmune response to acute binge ethanol withdrawal. J Neuroinflammation [Internet]. 2017 Dec 20;14(1):86. Available from: https://doi. org/10.1186/s12974-017-0856-z 